\section{On Hide's Magnetic Analogue of Ertel's Vorticity Theorem}

W. Schröder ${ }^{\mathrm{a}}$ and H.-J. Treder ${ }^{\mathrm{b}}$

a Hechelstr. 8, D-28777 Bremen-Roennenbeck

b Rosa-Luxemburg-Str. 17a, D-14482 Potsdam

Z. Naturforsch. 52 a, 210-211 (1997);

received January 8, 1997

The relativistic formulation of Hide's "magnetic analogue" of Ertel's potential vorticity theorem is Dirac's "new classical theory of electrons".

Key words: Hide's and Ertel's theorems; Potential vorticity; Potential magnetic field; Geomagnetism.

Dirac's "new classical theory of electrons" $[1,2]$ describes the relativistic hydrodynamics of conductive media with an "Ohm law"

$$
u^{\mu}=\frac{e}{m c}\left(A^{\mu}-g^{\mu v} \partial_{v} S\right), \quad(\mu, v=0,1,2,3) .
$$

In (1), $u^{\mu}=\frac{\mathrm{d} x^{\mu}}{\mathrm{d} t}$ is the four-dimensional velocity, $\mathrm{d} \tau$ the differential of the proper time, $A^{\mu}$ the four-dimensional electro-magnetic vector potential, and $S$ a gauge function. $g_{\mu v}$ means the metrical Minkowski tensor, and $e$ and $m$ are the charge and the rest mass of an electron, respectively.

According to (1), the norm of the velocity is

$$
-c^{2}=u_{\mu} u^{\mu}=\frac{e^{2}}{m^{2} c^{2}}\left(A_{\mu} A^{\mu}+g^{\mu v} \partial_{\mu} S \partial_{v} S-2 A^{\mu} \partial_{\mu} S\right) .
$$

The tensor of the four-dimensional rotation is

$\omega_{\mu v}=\partial_{v} u_{\mu}-\partial_{\mu} u_{v}=\frac{e}{m c}\left(\partial_{v} A_{\mu}-\partial_{\mu} A_{v}\right)=\frac{e}{m c} F_{\mu v}=-\omega_{v \mu}$,

where $F_{\mu v}$ is the anti-symmetric Maxwellian tensor of the electro-magnetic field.

The four-dimensional relativistic generalization of Helmholtz' theorem of the conservation of vorticity

Reprint requests to W. Schröder. means $[3-5]$

$$
\begin{aligned}
\mathrm{d} \omega_{\alpha \beta}= & u^{i} \partial_{j} \omega_{\alpha \beta}=\omega^{i}{ }_{\alpha} \partial_{\beta} u_{j}-\omega^{i}{ }_{\beta} \partial_{\alpha} u_{j} \\
& +\partial_{\beta} \frac{\mathrm{d} u_{\alpha}}{\mathrm{d} \tau}-\partial_{\alpha} \frac{\mathrm{d} u_{\beta}}{\mathrm{d} \tau}
\end{aligned}
$$

where $\frac{\mathrm{d} \Phi}{\mathrm{d} \tau}=u^{i} \partial_{j} \Phi$

Because of the identity $\frac{\mathrm{d}}{\mathrm{d} \tau} \mathrm{d} x^{\alpha}=u^{i} \partial_{j} \mathrm{~d} x^{\alpha}$ one can write (4), with the help of the Cartan exterior differential [6]

$$
\left[\mathrm{d} x^{\alpha} \wedge \mathrm{d} x^{\beta}\right]=-\left[\mathrm{d} x^{\beta} \wedge \mathrm{d} x^{\alpha}\right],
$$

like a relativistic formulation of Ertel's vorticity theorem [7]; see also [8]

$$
\frac{\mathrm{d}}{\mathrm{d} \tau}\left(\omega_{\mu v}\left[\mathrm{~d} x^{\mu} \wedge \mathrm{d} x^{v}\right]\right)=2 \partial_{v} \frac{\mathrm{d} u_{\mu}}{\mathrm{d} \tau}\left[\mathrm{d} x^{\mu} \wedge \mathrm{d} x^{v}\right] .
$$

By the Cartan-Stokes integral theorem, (5) yields the circulation theorem [6]

$$
\frac{\mathrm{d}}{\mathrm{d} \tau} \int_{C_{2}} \omega_{\mu v}\left[\mathrm{~d} x^{\mu} \wedge \mathrm{d} x^{\nu}\right]=2 \oint_{C_{1}} \frac{\mathrm{d} u_{\mu}}{\mathrm{d} \tau} \mathrm{d} x^{\mu}
$$

(with $C_{1}=\partial C_{2}$ ).

According to Poincaré's rules of exterior differentiation, $(5,6)$ with Dirac's law (1) yield an analogous equation for the electromagnetic field strength $F_{\mu v}=\partial_{v} A_{\mu}-\partial_{\mu} A_{v}$, namely the non-holonomic expression

$$
\frac{\mathrm{d}}{\mathrm{d} \tau}\left(F_{\mu v}\left[A^{\mu} \wedge A^{v}\right]\right)=2 \partial_{v} \frac{\mathrm{d} A_{\mu}}{\mathrm{d} \tau}\left[A^{\mu} \wedge A^{v}\right],
$$

together with a circulation theorem of the electromagnetic field

$$
\frac{\mathrm{d}}{\mathrm{d} \tau} \int_{C_{2}} F_{\mu \nu}\left[\mathrm{d} x^{\mu} \wedge \mathrm{d} x^{\nu}\right]=2 \oint_{C_{1}} \frac{\mathrm{d} A_{\mu}}{\mathrm{d} \tau} \mathrm{d} x^{\mu}
$$

according to the holonomic equations

$$
\frac{\mathrm{d}}{\mathrm{d} \tau}\left(F_{\mu v}\left[\mathrm{~d} x^{\mu} \wedge \mathrm{d} x^{v}\right]\right)=2 \partial_{v} \frac{\mathrm{d} A_{\mu}}{\mathrm{d} \tau}\left[\mathrm{d} x^{\mu} \wedge \mathrm{d} x^{v}\right] .
$$

These equations are a relativistic generalization of Hide's magnetic analogue [9, 10] of Ertel's vorticity theorem. The relativistic point of view generalizes the "magnetic analogue" of Hide to an "electro-magnetic" analogue. 
[1] P. A. M. Dirac, Proc. Roy. Soc. London A 209, 291 (1951).

[2] P. A. M. Dirac, Proc. Roy. Soc. London A 212, 330 (1951).

[3] H. Ertel, Ein System von Identitäten und seine Anwendung zur Transformation von Wirbelgleichungen der Hydrodynamik, Monatsberichte Deutsche Akad. Wiss. Berlin 4, 292 (1962).

[4] H. Ertel, Analogia entre las ecuaciones del movimimento y las ecuaciones del torbellino en la Hidrodinamica. Gerlands Beitr. Geophysik 72, 312 (1963)

[5] H.-J. Treder, Die allgemein-kovariante, relativistische Verallgemeinerung des 1 . Helmholtzschen Wirbelsatzes, Gerlands Beiträge Geophysik 78, 436 (1969).
[6] A. Lichnerowicz, Relativistic hydrodynamics and magnetohydrodynamic, New York, 1967.

[7] H. Ertel, Meteorol. Zs. 59, 227 (1942) (engl. transl. in W. Schröder ed., Geophysical Hydrodynamics and Ertel's Potential Vorticity, Bremen 1991).

[8] H.-J. Treder, Zur allgemein-relativistischen Integralform der Helmholtzschen Wirbeltheoreme, Gerlands Beiträge Geophysik 79, 1 (1970).

[9] R. Hide, The magnetic analogue of Ertel's vorticity theorem, Ann. Geophys. 1, 59 (1983).

[10] R. Hide, Potential magnetic field and potential vorticity in magnetohydrodynamics, Geophys. Int. J. 125, F1 - F3 (1996). 
Nachdruck - auch auszugsweise - nur mit schriftlicher Genehmigung des Verlages gestattet Verantwortlich für den Inhalt: A. KLEMM

Satz und Druck: Konrad Triltsch, Würzburg 